\title{
Incidência de disfagia orofaríngea em pacientes com paralisia cerebral do tipo tetraparéticos espásticos institucionalizados
}

\author{
Incidence of oropharyngeal dysphagia in institutionalized patients \\ with spastic tetraparetic cerebral palsy
}

\author{
Carla Lucchi ${ }^{1}$, Carla Patrícia Frigério Flório² ${ }^{2}$ Carolina Castelli Silvério ${ }^{3}$, Thaís Maria dos Reis ${ }^{4}$
}

\begin{abstract}
RESUMO
Objetivo: Verificar a incidência de disfagia orofaríngea em pacientes com paralisia cerebral do tipo tetraparesia espástica institucionalizados, correlacionando os achados com a consistência alimentar ingerida e o tipo de hidratação. Métodos: Participaram do estudo retrospectivo 140 pacientes, com média de idade 28 anos. Foram levantados os seguintes dados do prontuário médico: comprometimento da deglutição, sendo utilizado o protocolo ROGS para classificação; via de alimentação e consistências alimentares; forma de hidratação. Resultados: Verificou-se presença de disfagia orofaríngea em diferentes graus, sendo encontrada a deglutição funcional na maioria dos pacientes (40\%). Dos pacientes com deglutição funcional e disfagia leve, a maioria, 65\% e 50\% respectivamente, recebem dieta na consistência pastosa. Daqueles com disfagia moderada, 66,7\% recebem dieta na consistência semi-líquida, e 94,7\% de pacientes com disfagia grave fazem uso de via alternativa de alimentação. Do total de pacientes, 63,6\% recebem líquido fino e 10,7\% recebem gelatina como forma de hidratação. Conclusão: A incidência de disfagia orofaríngea, somando seus diferentes graus de comprometimento, mostra-se alta em portadores de paralisia cerebral do tipo tetraparética espástica, apesar da deglutição funcional ser a mais encontrada. A consistência alimentar pastosa é mais presente nos pacientes classificados como deglutição funcional e disfagia leve. Os pacientes com disfagia grave fazem uso de via alternativa de alimentação em sua maioria. O uso do líquido fino é o mais encontrado como forma de hidratação na maioria dos pacientes, sendo este substituído pela gelatina, mais frequentemente conforme maior o comprometimento dadeglutição.
\end{abstract}

Descritores: Paralisia cerebral/complicações; Transtornos de deglutição/epidemiologia; Alimentação; Pacientes internados

\section{INTRODUÇÃO}

Os pacientes com paralisia cerebral (PC), devido ao comprometimento motor global decorrente da alteração neurológica, podem apresentar comprometimentos na função de deglutição, que vão desde alterações no preparo oral do alimento ${ }^{(1-4)}$, até a presença de sinais sugestivos de penetração e/ou aspiração laringotraqueal do alimento ${ }^{(1,5-8)}$.

A presença das disfagias orofaríngeas pode ocasionar

Trabalho realizado no setor de Fonoaudiologia da Associação Cruz Verde de São Paulo - São Paulo (SP), Brasil.

(1) Fonoaudióloga da Associação de Assistência à Criança Deficiente - AACD

- São Paulo (SP), Brasil.

(2) Fonoaudióloga da Associação Cruz Verde de São Paulo - São Paulo (SP), Brasil.

(3) Pós-graduanda em Distúrbios da Comunicação Humana: Campo Fonoaudiológico do Departamento de Fonoaudiologia da Universidade Federal de São Paulo - UNIFESP - São Paulo (SP), Brasil.

(4) Fonoaudióloga da Associação Cruz Verde de São Paulo - São Paulo (SP), Brasil.

Endereço para correspondência: Carla Lucchi. Av. Bosque da Saúde, 713/4, Vila Clementino, São Paulo - SP, CEP: 04142-091. E-mail: carlalucchi@yahoo.com.br

Recebido em: 21/2/2008; Aceito em: 1/2/2009 implicações significativas para o desenvolvimento global, nutrição, hidratação, e estabilidade clínica, principalmente no sistema respiratório ${ }^{(6,9-10)}$. Dessa forma, torna-se necessário o adequado diagnóstico e o conhecimento sobre tais alterações, podendo assim minimizar os impactos clínicos.

O levantamento da incidência da disfagia em pacientes com PC permite, não apenas o conhecimento da doença e suas complicações, mas também alertar a equipe médica da necessidade de um acompanhamento mais detalhado e periódico relacionado à deglutição nesses casos.

Pouca literatura tem sido verificada no levantamento da incidência da disfagia orofaríngea em indivíduos com PC em idade mais avançada, dificultando o conhecimento do comprometimento da deglutição com o desenvolvimento do paciente, levando em consideração as compensações adquiridas na deglutição, assim como os desequilíbrios causados por novas posturas e/ou esquema corporal.

Dessa maneira, o objetivo deste estudo é verificar a incidência de disfagia orofaríngea e o seu grau de comprometimento em pacientes com PC do tipo tetraparesia espástica institucionalizados, correlacionando os achados com a consistência alimentar ingerida e o tipo de hidratação. 


\section{MÉTODOS}

Participaram deste estudo retrospectivo 140 pacientes com PC tetraparesia espástica, sendo 79 pacientes do sexo feminino e 61 do sexo masculino, com média de idade de 28 anos, que se encontram internados na Associação Cruz Verde, na cidade de São Paulo. As idades dos pacientes variaram de cinco a 50 anos, sendo 33 pacientes na faixa etária de cinco a 18 anos, 77 pacientes na faixa dos 19 aos 40 anos e 30 pacientes com 41 anos ou mais.

Após aprovação do Comitê de Ética em Pesquisa da instituição (parecer emitido em 28 de novembro de 2007), foram consultadas as avaliações fonoaudiológicas da deglutição realizadas pelos sujeitos no período de fevereiro a abril de 2007, constantes em seus prontuários médicos, levantando os seguintes dados:

- comprometimento da deglutição, sendo utilizado para isso o protocolo ROGS ${ }^{(11)}$, classificando a deglutição como funcional, disfagia leve, disfagia moderada e disfagia grave.

- via de alimentação utilizada, sendo que nos casos que se alimentavam por via oral, também foram levantadas informações quanto à consistência alimentar ingerida, sendo estas: sólida (alimentos cozidos e sem alteração em sua estrutura, carne em pequenos pedaços), semi-sólida (alimentos também cozidos, porém com maior tempo de cozimento e maior quantidade de alimentos pastosos, carne moída); pastosa (alimentos batidos homogeneamente incluindo a carne, com o arroz muito cozido e em pequenos pedaços), pastosa com arroz batido (idem à dieta pastosa; porém o arroz também é liquidificado) e semi-líquida (todos os alimentos são liquidificados juntos, tornando a dieta pastosa fina e homogênea).

- forma de hidratação, no caso de alimentação por via oral, podendo ser líquidos finos ou gelatina.

$\mathrm{Na}$ análise dos resultados foi utilizado o teste do Quiquadrado para proporções, com intuito de verificar as possíveis diferenças entre os percentuais de cada grupo estudado, para cada variável de interesse. Fixou-se em 0,05 ou 5\% o nível de rejeição da hipótese de nulidade.

\section{RESULTADOS}

Os resultados relacionados ao comprometimento da deglutição (Tabela 1) demonstram presença de disfagia orofaríngea em diferentes graus, com percentuais muito próximos, sendo observado que o maior deles representa a presença de deglutição funcional, correspondendo a $28,57 \%$ do total, seguida pela disfagia grave, que representa $27,14 \%$ dos pacientes. A classificação de disfagia moderada foi a menos frequente com $21,43 \%$.

Tabela 1. Distribuição dos pacientes em relação do grau de comprometimento da deglutição

\begin{tabular}{lcc}
\hline Grupo & Frequência (N) & Percentual (\%) \\
\hline Deglutição funcional & 40 & 28,57 \\
Disfagia leve & 32 & 22,86 \\
Disfagia moderada & 30 & 21,43 \\
Disfagia grave & 38 & 27,14 \\
\hline Total & 140 & 100 \\
\hline
\end{tabular}

Com relação ao tipo de dieta, conforme pode ser observado na Tabela 2, a pastosa foi a mais frequente $(33,57 \%)$, seguida pela dieta semi-líquida $(29,29 \%)$. A presença de sonda nasoenteral (SNE) representou 26,43\% do total de pacientes. Analisando as consistências por classificação do comprometimento da deglutição, verifica-se que na deglutição funcional e na disfagia leve, a dieta de maior frequência foi pastosa, com $65 \%$ e $50 \%$, respectivamente. Na classificação de disfagia moderada, observa-se a dieta semi-líquida como a mais presente $(66,67 \%)$. A presença de SNE nos pacientes classificados como disfágicos graves encontra-se em $94,74 \%$ destes.

Com relação à significância estatística da presença das dietas, foi realizada comparando-se cada tipo de dieta (SNE, semi-líquida, pastosa com arroz batido, pastosa, semi-sólida e sólida) entre si, conforme o valor total entre todos os pacientes da pesquisa. Assim, observa-se que a presença de SNE, da dieta semi-líquida e da dieta pastosa apresentou diferenças estatisticamente significantes quando comparados seus valo-

Tabela 2. Distribuição dos pacientes em relação do grau de comprometimento da deglutição e ao tipo de dieta

\begin{tabular}{|c|c|c|c|c|c|c|c|}
\hline \multicolumn{2}{|c|}{ Grupo deglutição } & \multirow{2}{*}{$\begin{array}{c}\text { SNE } \\
0\end{array}$} & \multirow{2}{*}{$\begin{array}{c}\text { Semi-líquida } \\
6\end{array}$} & \multirow{2}{*}{$\begin{array}{c}\text { Pastosa } \\
\text { (arroz batido) }\end{array}$} & \multirow{2}{*}{$\begin{array}{c}\text { Pastosa } \\
26\end{array}$} & \multirow{2}{*}{$\begin{array}{c}\text { Semi-sólida } \\
3\end{array}$} & \multirow{2}{*}{$\begin{array}{c}\text { Sólida } \\
3\end{array}$} \\
\hline Funcional & $\mathrm{N}$ & & & & & & \\
\hline & $\%$ & 0,00 & 15,00 & 10,00 & 65,00 & 2,63 & 7,50 \\
\hline \multirow[t]{2}{*}{ Leve } & $\mathrm{N}$ & 1 & 13 & 1 & 16 & 1 & 0 \\
\hline & $\%$ & 3,13 & 40,63 & 3,13 & 50,00 & 3,13 & 0,00 \\
\hline \multirow[t]{2}{*}{ Moderada } & $\mathrm{N}$ & 0 & 20 & 5 & 5 & 0 & 0 \\
\hline & $\%$ & 0,00 & 66,67 & 16,67 & 16,67 & 0,00 & 0,00 \\
\hline \multirow[t]{2}{*}{ Grave } & $\mathrm{N}$ & 36 & 2 & 0 & 0 & 0 & 0 \\
\hline & $\%$ & 94,74 & 5,26 & 0,00 & 0,00 & 0,00 & 0,00 \\
\hline \multirow[t]{3}{*}{ Total } & $\mathrm{N}$ & 37 & 41 & 10 & 47 & 2 & 3 \\
\hline & $\%$ & 26,43 & 29,29 & 7,14 & 33,57 & 1,45 & 2,14 \\
\hline & Valor de $p$ & $<0,001^{\star}$ & $0,005^{\star}$ & 0,878 & $0,002^{*}$ & 0,999 & 0,915 \\
\hline
\end{tabular}

Legenda: $\mathrm{SNE}=$ sonda nasoenteral; $\mathrm{N}=$ número de sujeitos. * Diferença estatisticamente significante $(p \leq 0,05)$ 
res. Dessa maneira, com objetivo de analisar na comparação, em quais grupos esta diferença estatisticamente significante encontrava-se presente, para cada variável (SNE, dieta semilíquida e dieta pastosa), foi realizada a comparação par a par entre os grupos estudados (Tabela 3). Dessa forma, pode-se verificar que a diferença estatisticamente significante da presença de SNE entre os grupos, na comparação par a par, ocorreu na comparação deglutição funcional x disfagia grave, disfagia leve $\mathrm{x}$ disfagia grave e disfagia moderada $\mathrm{x}$ disfagia grave, ou seja, na comparação do grupo disfagia grave com os demais. No caso da dieta semi-líquida, a significância estatística ocorreu na comparação entre os grupos disfagia moderada $\mathrm{x}$ disfagia grave. $\mathrm{E}$, na análise da dieta pastosa, a diferença estatisticamente significante esteve presente na comparação entre os grupos deglutição funcional $x$ disfagia grave e disfagia leve $\mathrm{x}$ disfagia grave.

Tabela 3. Comparação entre os grupos para cada variável considerada (valores de $p$ )

\begin{tabular}{lccc}
\hline Par de grupos & SNE & Semi-líquida & Pastosa \\
\hline Funcional X grave & $<0,001^{*}$ & 0,648 & $<0,001^{*}$ \\
Funcional X leve & 0,900 & 0,213 & 0,325 \\
Funcional X moderada & $>0,999$ & $>0,999$ & $>0,999$ \\
Grave X leve & $<0,001^{*}$ & 0,103 & $0,021^{*}$ \\
Grave X moderada & $<0,001^{*}$ & $0,003^{*}$ & 0,500 \\
Leve X moderada & 0,900 & 0,134 & 0,105 \\
\hline
\end{tabular}

Legenda: $\mathrm{SNE}=$ sonda nasoenteral; $\mathrm{N}=$ número de sujeitos. ${ }^{*}$ Diferença estatisticamente significante $(p \leq 0,05)$

A Tabela 4 demonstra a distribuição entre os grupos de pacientes quanto ao tipo de hidratação. Nesta análise, o total de pacientes foi 104 ( $n=104)$, uma vez que se excluíram os 36 pacientes com disfagia grave que faziam uso de SNE como forma de hidratação. $\mathrm{O}$ único paciente com disfagia leve que fazia uso de SNE entrou na análise do tipo de hidratação, uma vez que recebia oferta de líquido fino por via oral. O uso de líquido fino como forma de hidratação foi o mais frequente,

Tabela 4. Distribuição dos pacientes em relação do grau de comprometimento da deglutição e ao tipo de hidratação

\begin{tabular}{lccc}
\hline Grupo deglutição & & Líquido fino & Gelatina \\
\hline Funcional & $\mathrm{N}$ & 40 & 0 \\
\multirow{2}{*}{ Leve } & $\%$ & 100,00 & 0,00 \\
& $\mathrm{~N}$ & 30 & 2 \\
Moderada & $\%$ & 93,75 & 6,25 \\
& $\mathrm{~N}$ & 19 & 11 \\
Grave & $\%$ & 63,33 & 36,67 \\
& $\mathrm{~N}$ & 0 & 2 \\
Total & $\%$ & 0,00 & 5,26 \\
& $\mathrm{~N}$ & 89 & 15 \\
& $\%$ & 63,57 & 10,71 \\
& Valor de $\mathrm{p}$ & $<0,001^{*}$ & 0,092 \\
\hline
\end{tabular}

Legenda: $\mathrm{N}=$ número de sujeitos. ${ }^{*}$ Diferença estatisticamente significante $(p \leq 0,05)$ com 63,57\%, havendo diferença estatisticamente significante quando comparado com a presença de gelatina $(10,71 \%)$ como forma de hidratação. Na Tabela 5, é realizada a comparação par a par entre os grupos, com relação à frequência do líquido como forma de hidratação, com objetivo de se verificar entre quais grupos esta diferença com significância estatística se mantém. Na comparação entre os grupos deglutição funcional $\mathrm{x}$ disfagia grave, disfagia grave $\mathrm{x}$ disfagia leve, e disfagia grave $\mathrm{x}$ disfagia moderada, é verificada diferença estatisticamente significante.

Tabela 5. Comparação entre os grupos para a variável Líquido (valores de $\mathrm{p}$ )

\begin{tabular}{lc}
\hline Par de grupos & Líquido \\
\hline Funcional X grave & $<0,001^{*}$ \\
Funcional X leve & 0,133 \\
Funcional X moderada & $>0,999$ \\
Grave X leve & $<0,001^{*}$ \\
Grave X moderada & $0,003^{*}$ \\
Leve X moderada & $0,010^{*}$ \\
\hline
\end{tabular}

* Diferença estatisticamente significante $(p \leq 0,05)$

\section{DISCUSSÃO}

Com relação ao comprometimento da deglutição, a classificação de deglutição funcional foi mais presente neste estudo. Tal fato pode ser decorrente dos mecanismos compensatórios adquiridos por estes indivíduos ao longo dos anos, de acordo com o que se observa na prática clínica, como por exemplo, a extensão cervical como auxílio na propulsão oral do bolo e as deglutições múltiplas, auxiliando na limpeza da estase em recessos faríngeos. Além disso, a atuação e principalmente as orientações fonoaudiológicas com relação à alimentação destes pacientes, com ênfase no que se refere ao tipo de consistência alimentar e ao melhor posicionamento para cada indivíduo, também podem ter contribuído para a maior funcionalidade no processo de deglutição.

A influência do posicionamento na alimentação é confirmada por outras pesquisas. $\mathrm{O}$ efeito do posicionamento na alimentação é imediato e a aspiração pode ser minimizada ou eliminada quando a posição da cabeça, pescoço e tronco são alinhadas com as habilidades motoras orais ${ }^{(6)}$. Outro estudo verificou que a flexão do pescoço em $30^{\circ}$ diminui a tendência de aspiração durante o processo de deglutição ${ }^{(12)}$. A adequação postural em crianças com paralisia cerebral favorece de forma significativa as funções de sucção, respiração e deglutição, além da coordenação entre elas, tornando a alimentação mais segura e eficiente ${ }^{(13)}$. Pesquisa realizada com 17 crianças com alterações sensório-motoras verificou adequação no processo de deglutição somente em $23,5 \%$ destas, sendo que este percentual elevou-se para $41,1 \%$, após as orientações quanto à postura alimentar ${ }^{(14)}$. $\mathrm{O}$ alinhamento postural adequado é importante no processo normal de alimentação. Crianças com paralisia cerebral frequentemente apresentam alterações no posicionamento, principalmente a extensão cervical, o que exacerba as dificuldades na deglutição ${ }^{(15)}$.

Apesar da maioria dos pacientes ter apresentado deglutição 
funcional, foi encontrado um alto índice de disfagia orofaríngea, se forem somados seus diferentes graus (leve, moderada e grave). Tal resultado concorda com estudos da literatura, que afirmam que os pacientes com paralisia cerebral, em decorrência de suas alterações posturais e sensório-motoras, apresentam alterações na função de deglutição, que vão desde comprometimentos no transporte do bolo alimentar para a faringe, até a presença de aspiração traqueal do alimento ${ }^{(1-4)}$.

Estudo $^{(9)}$ realizado em 32 crianças com PC tetraparética espástica aponta que todas apresentavam alterações na fase oral da deglutição e $40 \%$ apresentavam aspiração traqueal, sendo a consistência líquida a mais frequentemente aspirada. $\mathrm{Na}$ interpretação desses dados, verifica-se que $100 \%$ das crianças apresentavam algum grau de disfagia, sendo que no estudo atual, este percentual foi de $71,43 \%$, e $40 \%$ dos pacientes apresentavam disfagia moderada ou grave, por apresentaram aspiração traqueal, valor similar ao estudo atual de 48,57\%. Em outro estudo ${ }^{(10)}$ observou-se que das 16 crianças com PC tetraparética espástica pesquisadas, $68,75 \%$ apresentaram prejuízos na fase oral da deglutição, sendo a consistência líquida aspirada em $12,5 \%$ dos casos. O percentual de pacientes com disfagia moderada ou grave $(12,5 \%)$ deste estudo pela presença de aspiração diferencia-se do estudo atual.

Das 35 crianças acompanhadas em um estudo ${ }^{(16)}, 60 \%$ destas apresentavam problemas alimentares frequentes, de acordo com seus pais, verificando os autores uma relação entre a presença das dificuldades alimentares e a severidade motora global. Tal fato condiz com os dados do estudo atual, pois o percentual de presença de algum grau de disfagia (60\%) assemelha-se ao encontrado $(71,43 \%)$. Outro estudo ${ }^{(17)}$ indicou $40 \%$ de prevalência de aspiração em crianças com paralisia cerebral, sendo a consistência alimentar líquida três vezes mais aspirada do que consistências mais sólidas. O percentual de aspiração deste estudo indica o percentual de prevalência de disfagia moderada ou grave, assemelhando-se ao de nosso estudo (48,57\%).

Estudo $^{(18)}$ realizado com 11 crianças com paralisia cerebral, sendo 5 com PC tetraparesia espástica e 6 com PC coreoatetóide, verificou a presença de aspiração traqueal através da videofluoroscopia da deglutição em $80 \%$ dos pacientes com PC tetraparesia espástica e em $67 \%$ dos pacientes com PC coreoatetóide.

Mesmo todos os pacientes deste estudo sendo do mesmo tipo clínico de paralisia cerebral, ou tetraparesia espástica, foram verificados diferentes níveis de comprometimentos na função de deglutição, uma vez que dentro de um mesmo tipo de paralisia cerebral existem diferenças quanto aos níveis motores globais, o que não foi levado em conta neste estudo.

A consistência alimentar mais encontrada neste estudo como indicada para os pacientes é a dieta pastosa, inclusive para aqueles pacientes classificados como apresentando deglutição funcional. Observa-se que crianças com paralisia cerebral apresentam limitações motoras globais, dentre estas a alteração no processo de deglutição e, mais especificamente, a alteração na mastigação, resultando em uma inabilidade em triturar os alimentos mais fibrosos, tornando a alimentação pastosa, a consistência mais indicada para suprir o aspecto nutricional nestes casos. Tal fato concorda com dados de literatura, que afirma ser a consistência alimentar pastosa a mais indicada para os pacientes que são incapazes de mastigar alimentos sólidos ${ }^{(12)}$. Em outro estudo ${ }^{(5)}$ foi observado que as crianças com PC e leve dificuldade alimentar mostraram redução da eficiência de mastigar alimentos de texturas sólidas, não ocorrendo o mesmo com outras texturas (viscosa, semi-sólida e pastosa), quando comparadas com um grupo controle de crianças sem alterações neurológicas. Assim, as consistências alimentares semi-sólida e sólida são pouco frequentes nos pacientes desta pesquisa, sendo vista em apenas $2,1 \%$ e $1,4 \%$ respectivamente do total de pacientes.

Relacionando a presença da via alternativa de alimentação (VA), um paciente com Disfagia Leve faz uso desta em decorrência de recusa alimentar por via oral. Somente dois pacientes com Disfagia Grave não apresentam a VA como forma de nutrição, demonstrando a impossibilidade da manutenção da via oral como forma de nutrição em pacientes com sinais de aspiração traqueal sem proteção espontânea das vias aéreas inferiores. Além disso, estes dois pacientes com Disfagia Grave e que fazem uso da alimentação por VO, na consistência semilíquida e com substituição do líquido fino por gelatina, são acompanhados frequentemente pelo setor de Fonoaudiologia da instituição, sendo a VA já indicada para um deles, porém devido à presença de deformidades ósseas e musculares, não é possível a realização de tal conduta.

Em relação ao tipo de ingestão hídrica, a maioria dos pacientes da pesquisa faz uso do líquido fino, sendo o uso da gelatina verificado com maior frequência nos pacientes com Disfagia Moderada e Grave, quando não estava presente a VA. Em dois pacientes com Disfagia Leve, a gelatina é encontrada como substituta do líquido fino, não por risco de aspiração traqueal, mas sim por um deles apresentar pouca aceitação para a consistência líquida, e pela presença de escape extraoral importante no outro, impossibilitando a hidratação por líquidos finos.

\section{CONCLUSÃo}

Os pacientes com paralisia cerebral do tipo tetraparesia espástica apresentam alta incidência de disfagia orofaríngea, somando seus diferentes graus de comprometimento. Em relação à hidratação, o líquido fino foi o mais utilizado nos pacientes que apresentam Deglutição Funcional e Disfagia Leve. A gelatina como forma de espessar o líquido fino foi encontrada nos pacientes classificados como o grupo de Disfagia Moderada. Alimentam-se em sua maioria de consistência pastosa, com grande parte dos pacientes com disfagia orofaríngea grave, fazendo uso de via alternativa de alimentação. 


\begin{abstract}
Purpose: To verify the incidence of oropharyngeal dysphagia in institutionalized patients with spastic tetraparetic cerebral palsy, correlating the findings with food consistency and type of hydration. Methods: A total of 140 patients, with an average of 28 years old, participated in the study. The following data were gathered from their medical records: swallowing impairment, as classified according to the ROGS protocol; feeding type and food consistencies; hydration type. Results: It was observed the presence of different degrees of oropharyngeal dysphagia, and most of the patients (40\%) had functional deglutition. From the patients with functional deglutition and mild dysphagia, the majority, $65 \%$ and $50 \%$ respectively, were put on a doughy-consistency-based diet. From the patients with moderate dysphagia, $66.7 \%$ were put on a semi-liquid-consistency-based diet, and $94.7 \%$ of the patients with severe dysphagia used alternative feeding means. From the total number of patients, $63.6 \%$ had a liquid diet, and $10.7 \%$ received gelatin as hydration. Conclusion: The incidence of oropharyngeal dysphagia, adding its different impairment degrees, was high among patients with spastic tetraparetic cerebral palsy, although functional deglutition was the most often found. Doughy consistency food was more common among patients diagnosed with functional deglutition and mild dysphagia. Most patients with severe dysphagia used alternative feeding means. Fine liquid was more often found as the hydration type for most patients, and it was frequently substituted by gelatin depending on the severity of the swallowing impairment
\end{abstract}

Keywords: Cerebral palsy/complications; Deglutition disorders/epidemiology; Feeding; Inpatients

\title{
REFERÊNCIAS
}

1. Gisel EG, Alphonce E. Classification of eating impairments based on eating efficiency in children with cerebral palsy. Dysphagia. 1995;10(4):268-74.

2. Yilmaz S, Basar P, Gisel EG. Assessment of feeding performance in patients with cerebral palsy. Int J Rehabil Res. 2004;27(4):325-9.

3. Rogers B. Feeding method and health outcomes of children with cerebral palsy. J Pediatr. 2004;145(2 Suppl):S28-32.

4. Odding E, Roebroeck ME, Stam HJ. The epidemiology of cerebral palsy: incidence, impairments and risk factors. Disabil Rehabil. 2006;28(4):183-91.

5. Gisel EG, Alphonce E, Ramsay M. Assessment of ingestive and oral praxis skills: children with cerebral palsy vs. controls. Dysphagia. 2000;15(4):236-44.

6. Gisel EG, Schwartz S, Petryk A, Clarke D, Haberfellner H. "Wole body" mobility after one year of intraoral appliance therapy in children with cerebral palsy and moderate eating impairment. Dysphagia. 2000;15(4):226-35.

7. Aurelio SR, Genaro KF, Macedo Filho ED. Análise comparativa dos padrões de deglutição de crianças com paralisia cerebral e crianças normais. Rev Bras Otorrinolaringol. 2002;68(2):167-73.

8. Glassburn DL, Deem JF. Thickener viscosity in dysphagia management: variability among speech-language pathologists. Dysphagia. 1998;13(4):218-22.

9. Furkim AM, Behlau MS, Weckx LLM. Avaliação clínica e videofluoroscópica da deglutição em crianças com paralisia cerebral tetraparética espástica. Arq Neuropsiquiatr. 2003;61(3A):611-6.

10. Wright RE, Wright FR, Carson CA. Videofluoroscopic assessment in children with severe cerebral palsy presenting with dysphagia. Pediatr Radiol. 1996;26(10):720-2.
11. Silva RG, Vieira MM. Disfagia orofaríngea neurogênica em adultos pós-acidente vascular encefálico: identificação e classificação. In: Macedo Filho E, Pisani JC, Carneiro J, Gomes G. Disfagia: abordagem multidisciplinar. 3a ed. São Paulo: Frôntis Editorial, 1999. p.29-46.

12. Larnert G, Ekberg O. Positioning improves the oral and pharyngeal swallowing function in children with cerebral palsy. Acta Paediatr. 1995;84(6):689-92.

13. Flabiano FC, Limongi SCO, Val DC, Silva KCL. Intervenção fonoaudiológica junto a mães de crianças com alterações sensóriomotoras: enfoque na adequação da postura corporal durante a sucção. Rev Soc Bras Fonoaudiol. 2005;10(2):77-82.

14. Val DC, Limongi SCO, Flabiano FC, Silva KCL. Sistema estomatognático e postura corporal na criança com alterações sensóriomotoras. Pró-Fono. 2005;17(3):345-54.

15. Redstone F, West JF. The importance of postural control for feeding. Pediatr Nurs. 2004;30(2):97-100.

16. Dahl M, Thommessen M, Rasmussen M, Selberg T. Feeding and nutritional characteristics in children with moderate or severe cerebral palsy. Acta Paediatr. 1996;85(6):697-701.

17. DeMatteo C, Matovich D, Hjartarson A. Comparison of clinical and videofluoroscopic evaluation of children with feeding and swallowing difficulties. Dev Med Child Neurol. 2005;47(3):149-57.

18. Silva AB, Piovesana AM, Barcelos IH, Capellini SA. Evaluación clínica y videofluoroscópica de la deglución en pacientes con parálisis cerebral tetraparésica espástica y atetósica. Rev Neurol. 2006;42(8):462-5. 